\title{
Оптимизация технологических параметров процесса сухого охмеления с разработкой методики интегральной оценки качества пива
}

\author{
Новикова Инна Владимировна \\ Воронежский государственный университет инженерных технологий \\ Адрес: 394036, Россия, г. Воронеж, пр-т Революции, д. 19 \\ E-mail:noviv@list.ru
}

Рукавицын Павел Владимирович

Воронежский государственный университет инженерных технологий Адрес: 394036, Россия, г. Воронеж, пр-т Революиии, д. 19 E-mail:kafedra-tbisp@mail.ru

Муравьев Александр Сергеевич

Воронежский государственный университет инженерных технологий, Адрес: 394036, Россия, г. Воронеж, пр-т Революиии, д. 19

E-mail:hntrun@mail.ru

\begin{abstract}
Развитие крафтовых сортов пива со сложными фруктовыми, цветочными и цитрусовыми тонами, поиск оригинального вкуса и возрождение старинных рецептур служат предпосылками для применения сухого охмеления предприятиями пивоваренной промышленности. Процесс сухого охмеления проводили динамическим способом на экспериментальной установке, включающей аппарат для охмеления, сетчатый фильтр, емкость брожения, насос. Использовали хмели Saphir и Chinook. Исследование включало определение физико-химических показатели пива: концентрация гумулинонов, изо- $\alpha$-кислот и $\alpha$-кислот; общее содержание полифенолов; $p H$; объемная плотность напитка. Показатели сенсорной оценки включали: интенсивность и качество аромата хмеля; полнота вкуса; качество горечи; общее предпочтение. Для аппроксимации результатов исследования использовали радиальные нейронные сети. Определены оптимальные технологические параметры процесса: хмель Saphire, длительность процесса 12 ч, температура $3,0^{\circ} \mathrm{C}$. При сухом охмелении хмелем Chinook оптимальная длительность процесса составляет 10,5 ч, оптимальная температура составляет $3,0^{\circ} \mathrm{C}$. При температурах процесса сухого охмеления ниже $3,0{ }^{\circ} \mathrm{C}$ критерий оптимизации получается незначительно ниже, чем для $3,0{ }^{\circ} \mathrm{C}$. При температурах 1,5 и $2,0^{\circ} \mathrm{C}$ оптимальная длительность процесса составляет 8,15 и 11,25 суток соответственно. Использование комбинации хмелей Saphire + Chinook не дает улучшение общего критерия оптимальности по сравнению с использованием только хмеля Saphire. При длительности процесса 4 ч удается достигнуть значений критерия оптимизации более 0,60 , однако только при длительности порядка 12 ч удается достигнуть критерия оптимизации более 0,65 .
\end{abstract}

Ключевые слова: хмель, нейронные сети, Saphire, Chinook, критерий оптимизации, сухое охмеление

\section{Введение}

Для распространенных сортов пива сейчас характерно высокое содержание ароматических компонентов, экстрагируемых из хмеля, наряду с нормируемым количеством горьких веществ (Leitao et al., 2011; Takoi et al., 2016; Walsh et al., 2018; Меледина, 2003). Развитие крафтовых сортов пива со сложными фруктовыми, цветочными и цитрусовыми тонами, поиск оригинального вкуса и возрождение старинных рецептур служат предпосылками для применения сухого охмеления различными предприятиями данного сегмента пищевой промышленности (Dresel et al., 2015; Schönberger \& Kostelecky, 2011; Гернет, 2017; Koростелев и др., 2020; Матвеева \& Титов, 2014; Федоренко \& Трэнкл, 2017). Сухое охмеление может стать источником ул учшения сенсорных характеристик пива с вероятностью потенциального увеличения содержания в пивоваренной продукции соединений, оказывающих благоприятное воздействие на организм человека, позволя- 
ет в большей степени раскрыть потенциал хмеля (Donaldson, Bamforth, \& Heymann, 2012; Barry, Muggah, McSweeney, \& Walker, 2018; Kippensberger, Hanke, Biendl, Stettner, \& Lagemann, 2014; Zanoli \& Zavatti, 2008).

Известны данные исследователей о специальных технологиях внесения хмеля и соответствующем оборудовании (Бородулин, Сафонова, Просин, \& Миленький, 2017; Матвеева, \& Титов, 2014; Матвеева, \& Титов, 2015; van Opstaele, de Rouck, de Clippeleer, de Cooman, 2010). Однако недостаточно раскрыты вопросы по оптимизации условий и продолжительности динамического процесса охмеления с помощью математического аппарата.

Цель исследования - выявление закономерностей при комбинировании сортов хмеля, внесении хмеля на различных этапах производства пива, оптимизация условий и продолжительности процесса охмеления с последующей модификацией установки для динамического сухого охмеления.

\section{Материалы и методы исследования}

\section{Материалы}

Объектом исследования являлось светлое пиво верхового брожения, плотность начального сусла 11 \%; содержание этанола 5,2 \%; pH 3,82.

Применяли два сорта хмеля - Saphir и Chinook урожая 2020 года с содержанием компонентов: Saphir ( $\alpha$-кислота 3,5 \%); Chinook ( $\alpha$-кислота $15,0 \%)$.

\section{Оборудование}

Процесс сухого охмеления проводили динамическим способом на экспериментальной установке, включающей аппарат для охмеления, сетчатый фильтр, емкость брожения, насос.

\section{Методы}

Кислоты хмеля и их производные: гумулиноны (гумулоны), изо- $\alpha$-кислоты, $\alpha$-кислоты, $\beta$-кислоты и их относительные концентрации в хмеле и пиве определяли с помощью метода высокоэффективной жидкостной хроматографии (ВЭЖХ) (Hao, et al., 2018; Haseleu, Intelmann, Hofmann, 2009; Чecнокова, 2011). Соединения выделяли из пива следующим образом: образец пива в количестве $5 \mathrm{~cm}^{3}$ подкисляли ортофосфорной кислотой $\left(100 \mathrm{~cm}^{3}\right)$ и экстрагировали изооктаном $\left(10 \mathrm{~cm}^{3}\right)$ в течение 30 мин. Экстракт переносили в стеклянную пробирку и выпаривали. К остатку после выпаривания добавляли ацетонитрил $\left(2 \mathrm{~cm}^{3}\right)$ и анализировали методом ВЭЖХ в трех повторностях. Для кислот хмеля и их производных были получены градуировочные графики на основе стандартов в диапазоне 1, 5, 10, 20, 40, 60 мг/дм ${ }^{3}$. Общее содержание полифенолов определяли спектрофотометрическим методом (Чеснокова, 2011).

Оценки состава и свойств исследуемых объектов осуществляли в соответствии с нормативной документацией: методы отбора проб - по ГОСТ 12786-80 ${ }^{1}$; определение спирта - по ГОСТ 12787$81^{2}$; определение экстрактивности начального сусла - по ГОСТ 12787-813; определение кислотности - по ГОСТ 12788-874; определение $\mathrm{pH}$ - по ГОСТ 31764-20125; определение цвета - по ГОСТ $12789-87^{6}$; определение органолептических показателей - по ГОСТ 30060-937.

\section{Процедура исследований}

В аппарат для сухого охмеления вносили гранулы хмеля (Saphir или Chinook, расход 100 г./гл), заполняли его пивом, после чего с помощью насоса проводили рециркуляцию суспензии. Продолжительность процесса до 8-10 часов. Во избежание вспенивания, температуру пива не поднимали выше $3{ }^{\circ} \mathrm{C}$; а разность давления между аппаратом и емкостью не превышала 0,2 атм (Новикова, Рукавицын, Муравьев, 2019).

\section{Анализ данных}

Для статистической оценки исследований применяли специальное ПО - Design Expert, v. 11.1.0, Stat-Ease Inc. и функции MS Excel. Экспериментальные данные обрабатывали вероятностными методами математической ста-

\footnotetext{
1 ГОСТ 12786-80. (2013). Пиво. Правила приемки и методы отбора проб. М.: Стандартинформ.

${ }^{2}$ ГОСТ 12787-80. (2013). Пиво. Методы определения спирта, действительного экстракта и расчет сухих веществ в начальном сусле. М.: Стандартинформ.

3 Там же.

${ }^{4}$ ГОСТ 12788-87. (2015). Пиво. Методы определения кислотности. М.: Стандартинформ.

5 ГОСТ 31764-2012. (2013). Пиво. Метод определения рН. М.: Стандартинформ.

6 ГОСТ 12789-87. (2011). Пиво. Методы определения цвета. М.: Стандартинформ.

7 ГОСТ 30060-93. (2013). Пиво. Методы определения органолептических показателей и объема продукции. М.: Стандартинформ.
} 
тистики - дисперсионным и корреляционным анализом (Грачев \& Плаксин, 2005).

Для аппроксимации зависимостей показателей от времени использовали радиальные нейронные сети (Bishop, 1995; Шелехова, Скворцова, Полтавская, 2021).

\section{Результаты и их обсуждение}

Полученные на этапе экспериментальных исследований результаты позволяют определить оптимальную длительность процесса охмеления, температуру и состав комбинации хмелей. Общий принцип оптимизации изображен схематично на Рисунке 1.

Факторами оптимизации являются: $t$ - длительность процесса охмеления; $T$ - температура процесса охмеления; с - концентрация хмеля Chinook в комбинации хмелей Saphir и Chinook (представляет собой вещественное число от 0 до 1).

Авторы данной статьи, в отличие от других исследователей, осуществили постановку задачи оптимизации технологических параметров процесса сухого охмеления с методикой прогнозирования, не используемой ранее, что позволяет для заданной комбинации хмелей Saphir и Chinook определить показатели сенсорной оценки в приближении линейной комбинации с учетом концентрации хмелей, физико-химические показатели пива, интегральную оценку качества пива. А также разработать математический аппарат - комбинацию линейной интерполяции и аппроксимации радиальной нейронной сетью, что позволит получить плавные функции при изменении времени, температуры или состава хмелей.

Разработанная и изложенная ниже методика прогноза позволяет определить следующие физико-химические показатели пива: $c_{\mathrm{r}}-$ концентрация гумулинонов; $\mathrm{c}_{\text {икк }}-$ концентрация изо- $\alpha$-кислот; $c_{\alpha \mathrm{K}}-$ концентрация $\alpha$-кислот; $\mathrm{c}_{\text {пф }}-$ общее содержание полифенолов; $p H$ - водородный показатель (показатель кислотности); $\rho$ - объемная плотность напитка.

Для заданной комбинации хмелей Saphir и Chinook можно определить показатели сенсорной оценки в приближении линейной комбинации с учетом концентрации хмелей: $И_{\text {ах }}$ - интенсивность аромата хмеля; $\mathrm{K}_{\mathrm{ax}}-$ качество аромата хмеля; $\Pi_{\text {в }}$ - полнота вкуса; $\mathrm{K}_{\mathrm{r}}$ - качество горечи; $\Pi_{\text {сз }}$ - предпочтение (среднее значение).

На основе спрогнозированных для данного набоpa $t, T, c$ физико-химических показателей и показателей сенсорной оценки определяются частные критерии оптимизации: $\mathrm{K}_{\text {фпп }}$ - критерий оптимизации физико-химических показателей; $\mathrm{K}_{\text {со }}$ - критерий оптимизации сенсорной оценки (Новикова и др., 2018).

На основе частных критериев оптимизации определяется общий критерий оптимизации $\mathrm{K}_{\text {оп }}$, который представляет собой интегральную оценку качества пива. Таким образом общий критерий оптимизации является функцией трех технологических параметров: $\mathrm{K}_{\text {оп }}(t, T, c)$.

Оптимизация заключается в определении значений или диапазонов (что лучше для практических

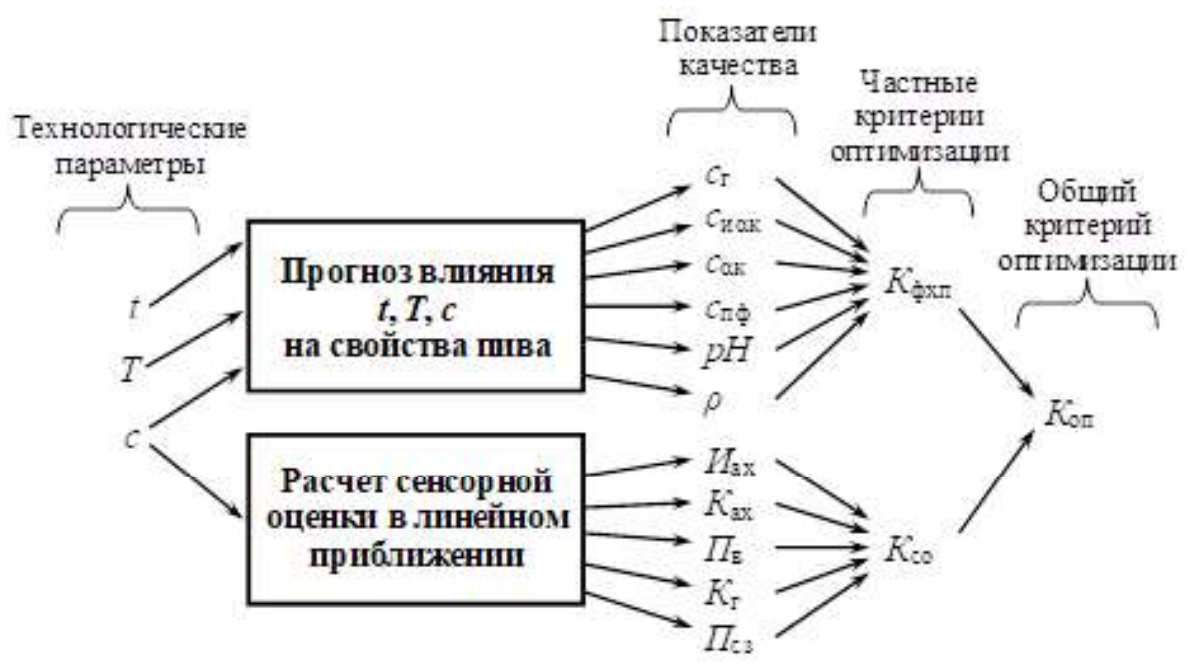

Рисунок 1. Постановка задачи оптимизации технологических параметров процесса сухого охмеления 
целей) параметров $t, T, c$, при которых функция принимает максимальное значение $\mathrm{K}_{\text {оп }}(t, T, c)$.

$$
K_{\text {on }}(t, T, c) \rightarrow \max \Rightarrow t_{\text {opt }}, T_{\text {opt }}, c_{\text {opt }},
$$

где $t_{\mathrm{opt}}, T_{\mathrm{opt}}, c_{\mathrm{opt}}-$ оптимальные значения технологических параметров.

Собранные в процессе экспериментального исследования данные позволяют получить функцию $\mathrm{K}_{\text {оп }}(t, T, c)$ в аналитическом виде на основе линейной и нейросетевой аппроксимаций. По известной функции $\mathrm{K}_{\text {оп }}(t, T, c)$ численным методом производится поиск максимума и определяются оптимальные параметры.

Составление общего критерия оптимизации из частных критериев

В соответствии с часто используемым подходом общий критерий оптимизации $\mathrm{K}_{\text {оп }}$ представляет собой линейную комбинацию двух частных критериев оптимизации $\mathrm{K}_{\text {фхп }}$ и $\mathrm{K}_{\text {со }}$ :

$$
K_{o n}=W_{\phi x n} \cdot K_{\phi x n}+W_{c o} \cdot K_{c o}
$$

где $W_{\text {фхп }}$ и $W_{\text {со }}$ - весовые коэффициенты, которые могут принимать вещественные значения от 0 до 1.

Для использования такой комбинации частные

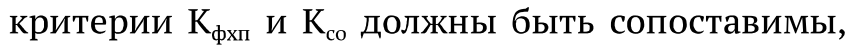
для чего ниже используются способы нормировки. Весовые коэффициенты отражают сравнительную важность физико-химической группы показателей и группы показателей сенсорной оценки в оценке качестве напитка. Весовые коэффициенты определены методом экспертных оценок и составляют $W_{\text {фхп }}=0,5, W_{\text {со }}=0,5$.

Частные критерии рассчитываются по шести $\left(\mathrm{K}_{\text {фхп }}\right)$ и по пяти $\left(\mathrm{K}_{\mathrm{co}}\right)$ показателям путем линейной комбинации показателей с весовыми коэффициентами и нормировкой на характерные значения показателей:

$$
K_{u}=\sum_{i=1}^{N_{P}} w_{i} \frac{1}{P_{i 2}-P_{i 1}}\left[\begin{array}{l}
P_{i}-P_{i 1}, d_{i}=1 \\
P_{i 2}-P_{i},
\end{array} d_{i}=-1,\right.
$$

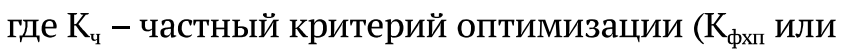
$\mathrm{K}_{\text {со }}$; $i$ - номер показателя; $N_{\mathrm{P}}$ - количество показателей; $w_{\mathrm{i}}-$ весовой коэффициент $i$-го показателя; $P_{\mathrm{i}}$ - показатели, на основе которых рассчитывается частный критерий оптимизации (с $\mathrm{c}_{\mathrm{r}}, \mathrm{c}_{\text {ихк }}$, И т. д.). $P_{\mathrm{i} 1}$ и $P_{\mathrm{i} 2}-$ минимальное и максимальное значения показателя $P_{\mathrm{i}}$ в экспериментальном исследовании; $d_{\mathrm{i}}-$ коэффициент благоприятной тенденции (равен 1 , если необходимо добиться максимума показателя и -1, если необходимо добиться минимума показателя).

Весовые коэффициенты $w_{\mathrm{i}}$, определенные экспертным путем и отражающие относительную важность показателей, приведены в Таблицах 1 и 2 (Коростелев и др., 2020). Сумма весовых коэффициентов в каждой группе равна 1.

\section{Аппроксимация экспериментальных данных}

Для определения оптимальных технологических параметров используются функции $K_{\mathrm{i}}(t, T, c)$ - зависимость физико-химических показателей $K_{\mathrm{i}}$

Таблица 1

Весовые коэффициенты физико-химических показателей

\begin{tabular}{lcccc}
\hline \multicolumn{1}{c}{ Показатель } & Обозначение & $\begin{array}{c}\text { Весовой коэффициент } w_{\mathrm{i}} \\
\text { (относительная важность) }\end{array}$ & $\begin{array}{c}\text { Оптималь- } \\
\text { ные значения }\end{array}$ & $\begin{array}{c}\text { Коэффициент благо- } \\
\text { приятной тенденции } d_{\mathrm{i}}\end{array}$ \\
\hline $\begin{array}{l}\text { Концентрация гу- } \\
\text { мулинонов }\end{array}$ & $\mathrm{c}_{\mathrm{r}}$ & 0,20 & $\max$ & 1 \\
$\begin{array}{l}\text { Концентрация } \\
\text { изо- } \alpha \text {-кислот }\end{array}$ & $\mathrm{c}_{\text {икк }}$ & 0,40 & $\min$ & -1 \\
$\begin{array}{l}\text { Концентра- } \\
\text { ция }- \text {-кислот }\end{array}$ & $\mathrm{c}_{\alpha \mathrm{k}}$ & 0,15 & $2,5 \pm 0,5$ & 0 \\
$\begin{array}{l}\text { Общее содержа- } \\
\text { ние полифенолов }\end{array}$ & $\mathrm{c}_{\mathrm{n}}$ & 0,15 & $\max$ & 1 \\
$\begin{array}{l}\text { Водородный по- } \\
\text { казатель }\end{array}$ & $\mathrm{pH}$ & 0,05 & $4,7 \pm 0,1$ & 0 \\
Плотность & - & 0,05 & $1,0045-1,0055$ & 0 \\
\hline
\end{tabular}


Таблица 2

Весовые коэффициенты показателей сенсорной оценки

\begin{tabular}{|c|c|c|c|c|}
\hline Показатель & Обозначение & $\begin{array}{c}\text { Весовой коэффици- } \\
\text { ент } w_{\mathrm{i}} \text { (относитель- } \\
\text { ная важность) }\end{array}$ & $\begin{array}{c}\text { Оптималь- } \\
\text { ные значения }\end{array}$ & $\begin{array}{c}\text { Коэффициент благо- } \\
\text { приятной тенденции } d_{\mathrm{i}}\end{array}$ \\
\hline $\begin{array}{l}\text { Интенсивность аро- } \\
\text { мата хмеля }\end{array}$ & $И_{\mathrm{ax}}$ & 0,10 & & 1 \\
\hline Качество аромата хмеля & $\mathrm{K}_{\mathrm{ax}}$ & 0,25 & & 1 \\
\hline Полнота вкуса & $\Pi_{\mathrm{B}}$ & 0,15 & $\max$ & 1 \\
\hline Качество горечи & $\mathrm{K}_{\mathrm{r}}$ & 0,25 & & 1 \\
\hline $\begin{array}{l}\text { Предпочтение (ср. знач.) } \\
\text { Общая оценка }\end{array}$ & $\Pi_{\mathrm{c} 3}$ & 0,25 & & 1 \\
\hline
\end{tabular}

(в частности, $\mathrm{c}_{\Gamma}, c_{\text {икк }}, \mathrm{c}_{\alpha \mathrm{\alpha}}, \mathrm{c}_{\text {пф }}, p H, \rho$ ) от длительности $t$ процесса сухого охмеления, температуры $T$, концентрации с хмеля Chinook в комбинации хмелей Saphire + Chinook.

Ранее в работе (Новикова, Рукавицын, Муравьев, 2018) представлены экспериментальные данные по зависимостям $K_{\mathrm{i}}(t, T, c)$. Для каждой функции получены 24 экспериментальные точки: для шести значений длительности процесса $t$ (0...12 ч), двух значений температуры $T\left(1,5\right.$ и 3,0 $\left.{ }^{\circ} \mathrm{C}\right)$ и двух значений концентрации $c$ (0 и 1 , то есть только хмель Saphire и только Chinook).

Так как 24 экспериментальные точки расположены сравнительно редко в пространстве факторов оптимизации $(t, T, c)$, было принято решение использовать аналитическую аппроксимацию для того, чтобы частные критерии оптимизации $K_{\mathrm{i}}(t, T, c)$ были непрерывными функциями в пространстве $(t, T, c)$.

Для построения аналитических функций $K_{\mathrm{i}}(t, T, c)$ используется линейная интерполяция по переменным $T$ и $c$, так как эти переменные в проведенном экспериментально исследовании принимали только два значения, а также аппроксимация радиальной нейронной сетью по переменной $t$, для которой экспериментально получено достаточно много (шесть) значений, позволяющих восстановить более детальную зависимость.

При расчете показателя $K_{\mathrm{i}}(t, T, c)$ в первую очередь производили линейную интерполяцию по переменной $c$, то есть по составу комбинированного хмеля:

$$
K_{i}(t, T)=K_{i}(t, T, 0)+c\left[K_{i}(t, T, 1) Ц K_{i}(t, T, 0)\right],
$$

где $K_{\mathrm{i}}(t, T)$ - показатель $i$ для заданной концентрации с в комбинации хмелей Saphir и Chinook; $K_{\mathrm{i}}(t$,
$T, 0)$ и $K_{\mathrm{i}}(t, T, 1)$ - показатель $i$ для хмелей Saphir и Chinook.

Далее производили линейную интерполяцию данных по температуре процесса T:

$K_{i}(t)=K_{i}(t, 1.5)+\frac{T Ц 1.5}{3.0 Ц 1.5}\left[K_{i}(t, 3.0) Ц K_{i}(t, 1.5)\right]$,

где 1,5 и 3,0 - значения температуры, при которых проводили эксперименты по сухому охмелению.

Для дальнейшего получения аналитической зависимости для функции $K_{\mathrm{i}}(t)$ (при заданном составе комбинации хмелей $c$ и температуре $T$ ) использовали радиальную нейронную сеть.

Радиальные нейронные сети более естественны для решения задач оптимизации, чем сигмоидальные, так как радиальные базисные функции хорошо воспроизводят локальные минимумы и максимумы (Осовский, 2004).

В радиальных функциях отсутствует необходимость использования большого количества скрытых слоев. Важное достоинство радиальных нейронных сетей - значительно упрощенный алгоритм обучения. Процесс обучения радиальной нейронной сети сводится к подбору центров и параметров формы базисных функций, а также подбору весов нейронов выходного слоя. При малом количестве исходных данных для обучения (в данной работе для построения аппроксимирующего выражения используется по шесть точек) обучение сводится к подбору только одного параметра $\sigma$ - параметра формы базисных функций (Осовский, 2004).

Для работы с радиальными нейронными сетями авторами разработана специальная компьютерная 
программа «Программа для прогнозирования результатов процесса сухого охмеления» на языке Object Pascal в среде программирования Delphi 7.

Пример аппроксимации показателя $c_{\text {икк }}(t)$ для сухого охмеления хмелем Chinook $(\mathrm{c}=1)$ при $1,5^{\circ} \mathrm{C}(T=$ $1,5)$ показан на Рисунке 2. График аппроксимирующей функции практически проходит близко к шести экспериментальным точкам $c_{\text {икј }}\left(t_{\mathrm{j}}\right)$, при этом кривая является плавной, начало и конец кривой не стремятся к бесконечности (как обычно происходит при полиномиальной аппроксимации).

С использованием формул и получены аппроксимирующие функции не только для зависимости показателя от времени $K_{\mathrm{i}}(t)$, но и от концентрации $c$ в комбинации хмелей и температуры процесса $T$, то есть полноценные функции $K_{\mathrm{i}}(t, T, c)$. Для примера на Рисунке 3 приведена серия графиков функций $c_{\mathrm{r}}\left(t, T=1,5^{\circ} \mathrm{C}, c\right)$.

Таким образом, разработанный математический аппарат - комбинация линейной интерполяции и аппроксимации радиальной нейронной сетью позволяют получить плавные функции при изме-

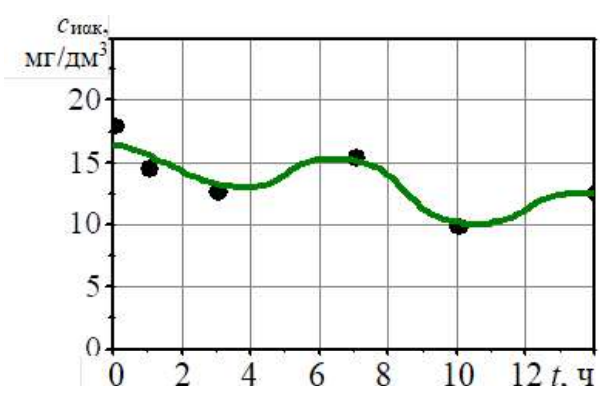

Рисунок 2. Пример аппроксимации экспериментальных данных радиальной нейронной сетью:

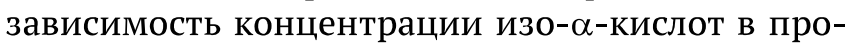
цессе сухого охмеления хмелем Chinook при $1,5^{\circ} \mathrm{C}$

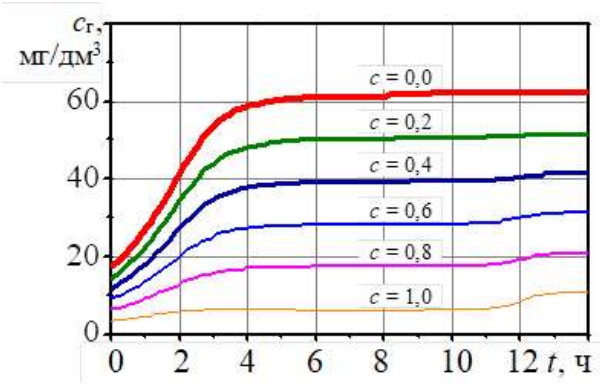

Рисунок 3. Зависимость концентрации гумулинонов $c_{\text {г }}$ от длительности процесса сухого охмеления при температуре $1,5^{\circ} \mathrm{C}$ при использовании различных комбинаций хмелей 1-c Saphire $+c$ Chinook нении времени, температуры или состава хмелей, что позволяет их использовать для дальнейшего решения общей задачи оптимизации.

\section{Решение общей задачи оптимизации}

Разработанный выше математический аппарат позволяет определить общий критерий оптимизации $K_{\text {оп }}(t, T, c)$ для различных комбинаций параметров $t$, $T, c$. Основной задачей оптимизации является поиск такой комбинации параметров $t, T, c$, при которой

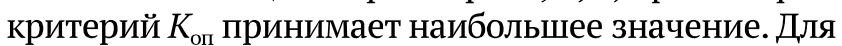

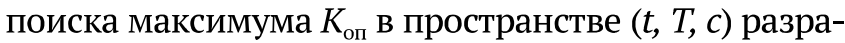
ботана компьютерная программа на языке Object Pascal в среде программирования Delphi 7. В про-

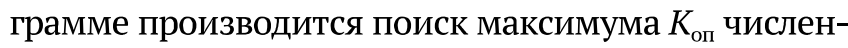
ным методом - методом упорядоченного перебора с равным шагом по трем переменным. Время поиска составляет около 2 с машинного времени. По каждой переменной исследуемый диапазон разбили на 101 уровень (переменная цикла изменяется от 0 до 100). В ходе перебора переменная $t$ изменяется от 0 до 12 часов с шагом 0,5 ч.; переменная $T$ изменяется от 1,5 до $3,0{ }^{\circ} \mathrm{C}$ с шагом $0,15{ }^{\circ} \mathrm{C}$; переменная с изменяется от 0,00 до 1,00 с шагом 0,01.

Оптимальная точка обычно может располагаться либо внутри исследуемой области факторного пространства, либо на границе. В данном случае точка с максимальным общим критерием $K_{\text {оп }}=$ $=0,679$ расположена на границе исследуемой области: $t=12$ ч; $T=3,0^{\circ} \mathrm{C} ; \mathbf{c}=0$. Таким образом, наилучшие физико-химические показатели и показатели сенсорной оценки достигаются при сухом охмелении хмелем Saphire в течении 12 ч при температуре $3,0{ }^{\circ} \mathrm{C}$.

Для более детального анализа поверхности отклика построены три серии картограмм (Рисунок 4). На картограммах отмечена точка оптимума обозначением «орt».

Использование комбинации хмелей Saphire + Chinook не дает улучшение общего критерия оптимизации по сравнению с использованием только хмеля Saphire (исчезает красная и оранжевая области на Рисунок 4, б). Однако, если необходимо использовать только хмель Chinook, оптимальная длительность процесса составляет 10,5 ч (точка лежит уже не на границе диапазона); оптимальная температура составляет $3,0^{\circ} \mathrm{C}$, однако при любых температурах критерий оптимизации практически одинаков и составляет 0,514 (Рисунок 4, в).

При температурах ниже $3,0{ }^{\circ} \mathrm{C}$ критерий оптимизации получается незначительно ниже, чем для 
$3,0^{\circ} \mathrm{C}$ (Рисунок 5). Так, при температурах 1,5 и $2,0^{\circ} \mathrm{C}$ удается достигнуть максимального критерия оптимизации 0,623 и 0,638 соответственно при длительности процесса 8,15 (Рисунок 5, а) и 11,25 (Рисунок 5,6 ) часов соответственно.

Аналогичные исследования других авторов показали важность контроля температуры пива при проведении сухого охмеления (Titus, et al., 2021; Матвеева \& Титов, 2015).

Даже при небольшой длительности процесса 4 часов удается достигнуть значений критерия оптимизации более 0,60 (оранжевая область на Рисунке 6, а). Однако только при длительности процесса порядка 12 часов удается достигнуть критерия оптимизации более 0,65 (красная область на Рисунке 6, в).

С точки зрения экономической эффективности желательно, чтобы длительность процесса сухого охмеления была минимальной при высоких показателях качества (Cibaka, et al., 2018; Maye, Smith, Leker, 2016; Матвеева \& Титов, 2015). Поэтому более подробно изучено изменение с течением времени $t$ критерия оптимизации $K_{\text {оп }}$, а именно, максимального значения $K_{\text {оп }}{ }^{\mathrm{m}}(t)$ среди различных температур ( $T$ от 0 до $20^{\circ} \mathrm{C}$ ) и различных концентраций комбинации хмелей (с от 0 до 1).

На зависимости критерия оптимизации от времени можно выделить три характерные точки
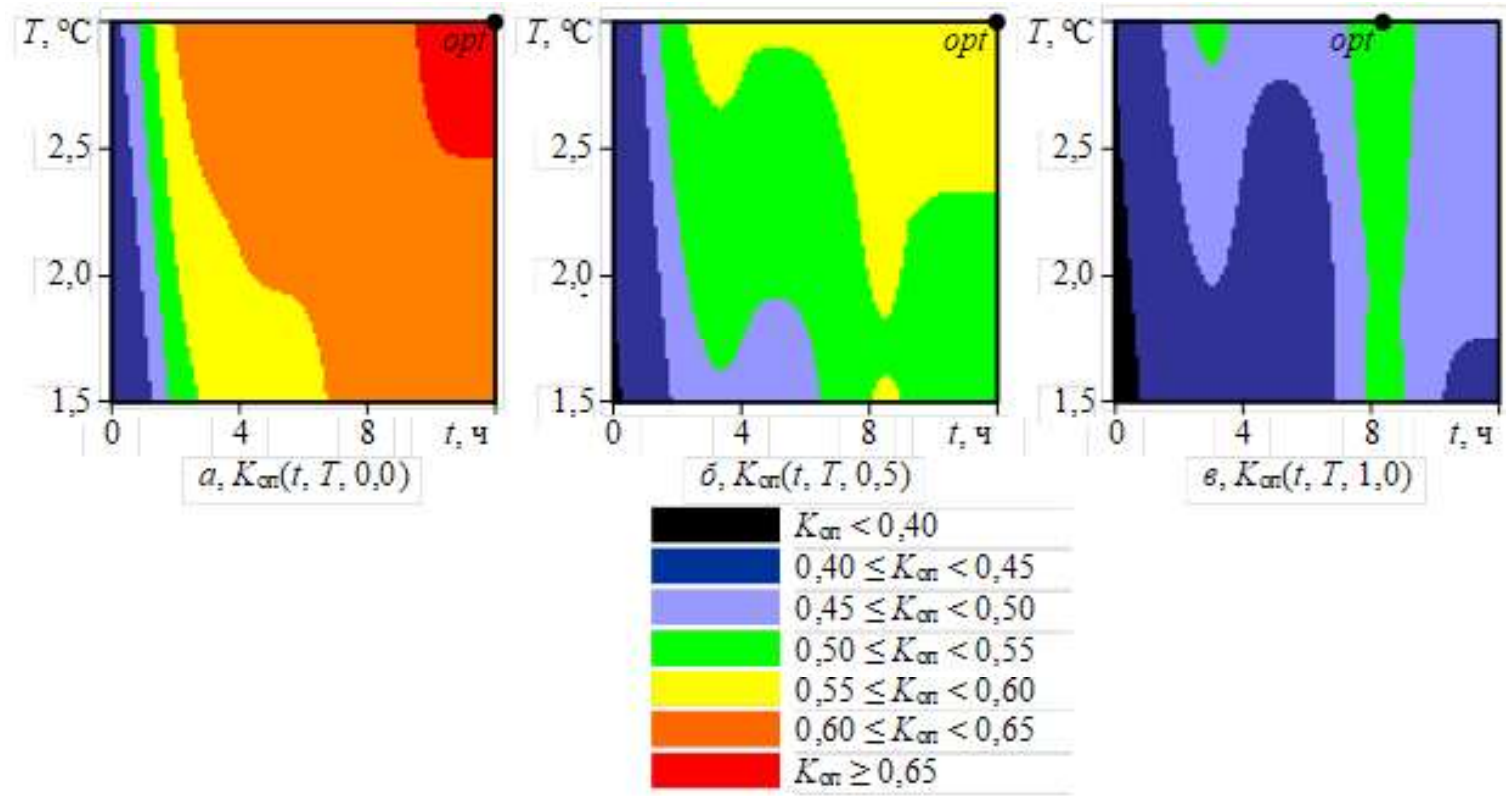

Рисунок 4. Картограммы оптимизации $K_{\text {оп }}(t, T)$ при разных концентрациях $c$ хмеля Chinook в комбинации хмелей $(-1 c)$ Saphire $+c$ Chinook
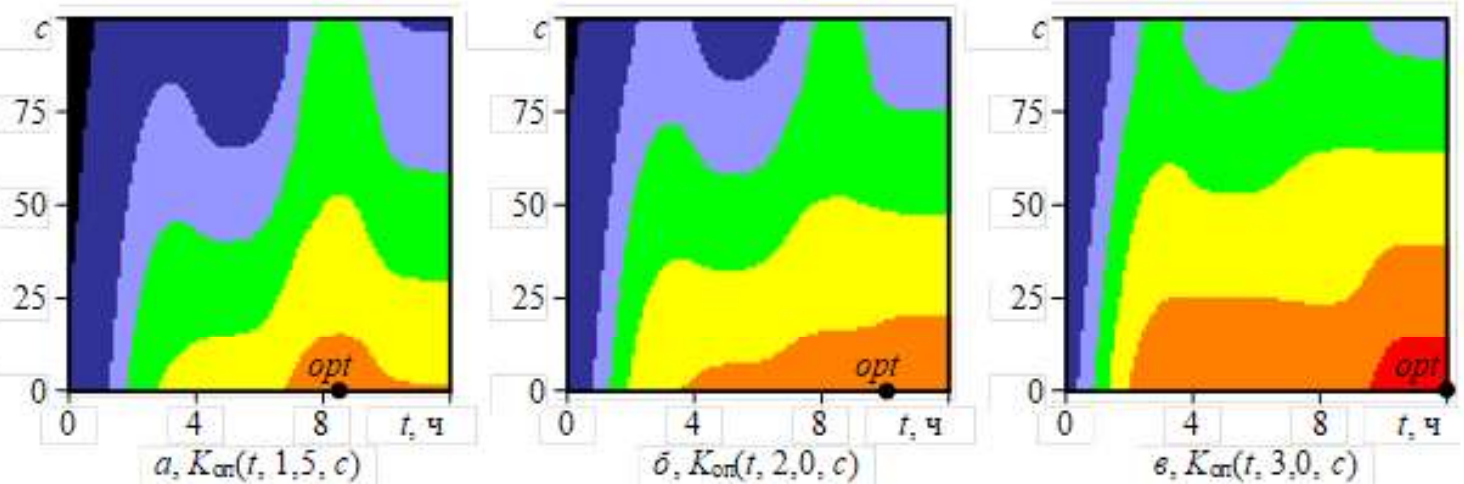

Рисунок 5. Картограммы оптимизации $K_{\text {оп }}(t, c)$ при разных температурах $T$ процесса сухого охмеления 

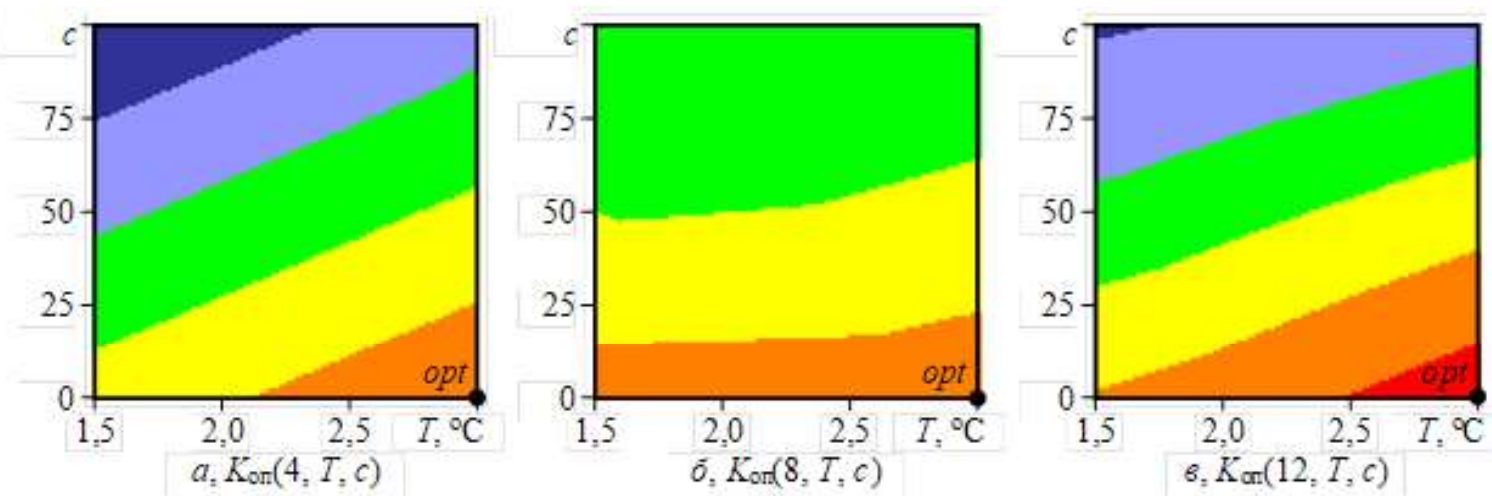

Рисунок 6. Картограммы оптимизации $K_{\text {оп }}(T, c)$ при разной длительности $t$ процесса сухого охмеления

(Рисунок 7). Точка А - это точка наименьшей длительности процесса 3,8 ч, при которой достигается высокие показатели качества пива - критерий оптимизации равен 0,636. Благодаря такой малой длительности процесса может быть достигнут наибольший экономический эффект.

Вторая характерная точка $B$ представляет собой локальную точку максимума и соответствует примерно вдвое большей длительности процесса 6,2 ч, при этом достигается больший критерий оптимизации 0,650 . С точки зрения экономического эффекта в данном случае необходимо оценивать баланс между снижением производительности в 1,75 раза процесса и повышением качества на $2,2 \%$. Данный вариант не позволяет получить пиво заметно высокого качества, при высокой длительности, поэтому не рекомендуется для реального производства.

Третья характерная точка C соответствует глобальному максимуму $K_{\text {оп }}{ }^{\mathrm{M}}=0,679$ и требует значительной длительности процесса 13,1 ч. В таком технологическом процессе получается наиболее качественное пиво (на 6,8 \% качественнее, чем для

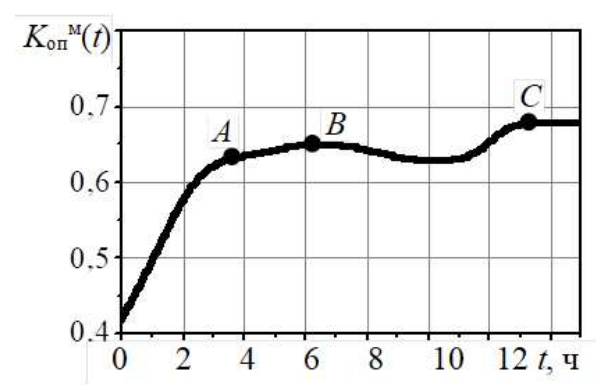

Рисунок 7. Зависимость от времени $t$ критерия оптимизации $K_{\text {оп }}{ }^{\text {, }}$ максимального в диапазонах температур $T$ от 1,5 до $3,0^{\circ}$ и концентраций с 0-1 быстрого варианта A), однако длительность процесса существенно выше (в 3,7 раза по сравнению с вариантом А). Поэтому с точки зрения экономической эффективности такой вариант приемлем для производства пива премиального класса.

Таким образом, разработана методика интегральной оценки качества пива, получаемого в процессе сухого охмеления, позволившая определить оптимальные технологические параметры процесса, исследовать влияние длительности процесса, температуры и комбинации хмелей на показатели качества, а также оценить экономическую эффективность трех вариантов длительности процесса.

\section{Выводы}

Разработан математический аппарат - комбинация линейной интерполяции и аппроксимации радиальной нейронной сетью - для получения аналитических непрерывных дифференцируемых функций показателей эффективности процесса охмеления от времени, температуры и состава хмелей, что позволяет их использовать для решения общей задачи оптимизации.

Определены оптимальные технологические параметры процесса сухого охмеления: хмель Saphire, длительность процесса 12 ч, температура $3,0^{\circ} \mathrm{C}$. Использование комбинации хмелей Saphire + Chinook не дает улучшение общего критерия оптимальности по сравнению с использованием только хмеля Saphire. При сухом охмелении хмелем Chinook oптимальная длительность процесса составляет 10,5 ч, оптимальная температура составляет $3,0^{\circ} \mathrm{C}$.

При температурах процесса сухого охмеления ниже $3,0^{\circ} \mathrm{C}$ критерий оптимизации получает- 
ся незначительно ниже, чем для $3,0^{\circ} \mathrm{C}$. При температурах 1,5 и $2,0^{\circ} \mathrm{C}$ оптимальная длительность процесса составляет 8,15 и 11,25 суток соответственно. Уже при небольшой длительности процесса 4 ч удается достигнуть значений критерия оптимизации более 0,60 , однако только при длительности порядка 12 ч удается достигнуть критерия оптимизации более 0,65 .

С точки зрения экономической эффективности возможны два варианта процесса сухого охмеления. Для получения пива обычного класса с достаточно высоким показателем качества 0,636 длительность процесса должна составлять 3,8 ч. Для получения пива премиального класса с показателем качества 0,679 (на 6,8 \% качественнее, чем обычного класса) длительность процесса должна составлять 13,1 ч.

\section{Литература}

Бородулин, Д. М., Сафонова, Е. А., Просин, М. В., \& Миленький, И. О. (2017). Исследование процесса охмеления пивного сусла с применением современного оборудования. Современные материалы, техника и технологии, 3 (11), 16-21.

Гернет, М. В. (2017). Перспективы расширения ассортимента напитков брожения для пивоваренных заводов малой мощности. Пиво и напитки, 3, 14-17.

Грачев, Ю. П., \& Плаксин, Ю. М. (2005). Математические методы планирования экспериментов. М.: ДеЛи Принт.

Коростелев, А. В., Рукавицын, П. В., Новикова, И. В., Кучменко, Т. А., Умарханов, Р. У., \& Муравьев, А. С. (2020). Исследование ароматобразующих компонентов хмеля с применением химических сенсоров. Известия Вузов. Прикладная Химия и Биотехнология, 10(3), 479-486. https:// doi.org/10.21285/2227-2925-2020-10-3-479-486

Матвеева, Н. А., \& Титов, А. А. (2014). Выбор сорта хмеля для технологии сухого охмеления. Научный журнал НИУ ИТМО. Процессы и аппараты пищевых производств, 4, 120-125.

Матвеева, Н. А., \& Титов, А. А. (2015). Применение технологии сухого охмеления в пивоварении. Научный журнал НИУ ИТМО. Процессы и аппараты пищевых производств, 1, 111-118.

Меледина, Т. В. (2003). Сырье и вспомогательные материалы в пивоварении. СПб.: Профессия.

Новикова, И. В., Агафонов, Г. В., Коротких, Е. А., Коростелев, А. В., Рукавицын, П. В., \& Новиков, С. В. (2018). Разработка методики проектирования безалкогольных напитков с учетом сбалансированности состава. Пиво и напитки, 2, 26-30.
Новикова, И. В., Рукавицын, П. В., \& Муравьев, А. С. (2018). Влияние основных параметров процесса сухого охмеления на физико-химические показатели пива. Технология и товароведение инновационных пищевых продуктов, 6, 9-17.

Новикова, И. В., Рукавицын, П. В., \& Муравьев, А. С. (2019). К вопросу перехода ароматических соединений хмеля в пиво при реализации сухого охмеления. Пищевая промышленность, 1, 69-73.

Осовский, С. (2004). Нейронные сети для обработки информации. М.: Финансы и статистика.

Федоренко, Б. Н., \& Трэнкл, Ф. (2017). Инновационное технологическое оборудование для крафтового пивоварения. Пиво и напитки, 1, 22-24.

Чеснокова, А. Н. (2011). Фенольные соединения хмеля обыкновенного (Humulus Lupulus L.) и хмелепродуктов [Кандидатская диссертация, Иркутский государственный технический университет]. Иркутск, Россия.

Шелехова, Н. В., Шелехова, Т. М., Скворцова, Л. И., \& Полтавская, Н. В. (2021). Идентификация спиртных напитков с применением искусственных нейронных сетей. Пищевая промышленность, 3, 4348. https://doi.org/10.24412/0235-2486-2021-3-0028

Barry, S., Muggah, E. M., McSweeney, M. B., \& Walker, S. (2018). A preliminary investigation into differences in hops' aroma attributes. International Journal of Food Science \& Technology, 53(3), 804811. https://doi.org/10.1111/ijfs.13656

Bishop, C. M. (1995). Neural networks for pattern recognition. Clarendon Press.

Cibaka, M.-L. K., Ferreira, C. S., Decourrière, L., Lorenzo-Alonso, C.-J., Bodart, E., \& Collin, S. (2018). Dry hopping with the dual-purpose varieties amarillo, citra, hallertau blanc, mosaic, and sorachi ace: Minor contribution of hop terpenol glucosides to beer flavors. Journal of the American Society of Brewing Chemists, 75(2), 122129. https://doi.org/10.1094/ASBCJ-2017-2257-01

Donaldson, B. R., Bamforth, C. W., \& Heymann, H. (2012). Sensory descriptive analysis and freechoice profiling of thirteen hop varieties as whole cones and after dry hopping of beer. Journal of the American Society of Brewing Chemists, 70(3), 176181. https://doi.org/10.1094/ASBCJ-2012-0710-01

Dresel, M., Praet, T., van Opstaele, F., van Holle, A., Naudts, D., Keukeleire, D. de, Cooman, L. de, \& Aerts, G. (2015). Comparison of the analytical profiles of volatiles in single-hopped worts and beers as a function of the hop variety. BrewingScience, 68(1-2), 8-28.

Hao, J., Dong, J., Yin, H., Yan, P., Ting, P. L., Li, Q., Tao, X., Yu, J., Chen, H., \& Li, M. (2018). Optimum method of analyzing hop derived aroma compounds in beer by headspace solid-phase microextraction (SPME) with GC/MS and their evolutions during chinese 
lager brewing process. Journal of the American Society of Brewing Chemists, 72(4), 261-270. https://doi. org/10.1094/ASBCJ-2014-1021-01

Haseleu, G., Intelmann, D., \& Hofmann, T. (2009). Identification and RP-HPLC-ESI-MS/MS quantitation of bitter-tasting beta-acid transformation products in beer. Journal of Agricultural and Food Chemistry, 57(16), 7480-7489. https://doi. org/10.1021/jf901759y

Kippensberger, M., Hanke, S., Biendl, M., Stettner, G. \& Lagemann, A. (2014). Transfer of nitrate and various pesticides into beer during dry hopping. BrewingScience, 67, 1-9.

Leitao, C., Marchioni, E., Bergaentzlé, M., Zhao, M., Didierjean, L., Taidi, B., \& Ennahar, S. (2011). Effects of processing steps on the phenolic content and antioxidant activity of beer. Journal of Agricultural and Food Chemistry, 59(4), 1249-1255. https://doi.org/10.1021/jf104094c

Maye, J. P., Smith, R., \& Leker, J. (2016). Humulinone formation in hops and hop pellets and its implications for dry hopped beers. Technical Quarterly, 53(1), 23-27. https://doi.org/10.1094/TQ53-1-0227-01

Schönberger, C., \& Kostelecky, T. (2011). 125th Anniversary review. The role of hops in brewing. Journal of the Institute of Brewing, 117(3), 259-267. https://doi.org/10.1002/j.2050-0416.2011.tb00471.x
Takoi, K., Tokita, K., Sanekata, A., Usami, Y., Itoga, Y., Koie, K., Matsumoto, I., \& Nakayama, Y. (2016). Varietal difference of hop-derived flavour compounds in late-hopped/dry-hopped beers. BrewingScience, 69, 1-7.

Titus, B. M., Lerno, L. A., Beaver, J. W., Byrnes, N. K., Heymann, H., \& Oberholster, A. (2021). Impact of dry hopping on beer flavor stability. Foods, 10(6), 1264. https://doi.org/10.3390/foods10061264

Van Opstaele, F., de Rouck, G., de Clippeleer, J., de Cooman, L. (2010). Analytical and Sensory Assessment of Hoppy Aroma and Bitterness of Conventionally Hopped and Advanced Hopped Pilsner Beers. Journal of the Institute of Brewing, 116(4), 445-458. https://doi. org/10.1002/j.2050-0416.2010.tb00796.x

Walsh, D. B., O'Neal, S. D., George, A. E., Groenendale, D. P., Henderson, R. E., Groenendale, G. M., \& Hengel, M. J. (2018). Evaluation of pesticide residues from conventional, organic, and nontreated hops on conventionally hopped, late-hopped, and wet-hopped beers. Journal of the American Society of Brewing Chemists, 74(1), 53-56. https://doi.org/10.1094/ASBCJ-2016-1115-02

Zanoli, P., \& Zavatti, M. (2008). Pharmacognostic and pharmacological profile of Humulus lupulus L. Journal of Ethnopharmacology, 116(3), 383-396. https://doi.org/10.1016/j.jep.2008.01.011 


\title{
Optimization of Technological Parameters of the Dry Hopping Process With the Development of a Methodology for the Integral Assessment of the Quality of Beer
}

\author{
Inna V. Novikova \\ Voronezh State University of Engineer.ng Technologies \\ 19, Revolution Avenue, Voronezh, 394036, Russian Federation \\ E-mail:noviv@list.ru \\ Pavel V. Rukavitsyn \\ Voronezh State University of Engineering Technologies \\ 19, Revolution Avenue, Voronezh, 394036, Russian Federation \\ E-mail:kafedra-tbisp@mail.ru

\begin{abstract}
Alexander S. Muravyov
Voronezh State University of Engineering Technologies, 19, Revolution Avenue, Voronezh, 394036, Russian Federation
\end{abstract} \\ E-mail:hntrun@mail.ru
}

\begin{abstract}
The development of craft beers with complex fruit, floral and citrus tones, the search for an original taste and the revival of old recipes are the prerequisites for the use of dry-hoping in the brewing industry. The dry-hopping process was carried out dynamically in an experimental setup with a hop apparatus, screen filter, fermentation tank and pump. Saphir and Chinook hops were used. The study included determination of physical and chemical parameters of beer: concentration of humulinones, iso- $\alpha$-acids and $\alpha$-acids; total content of polyphenols; $\mathrm{pH}$; volume density. Sensory evaluation indicators included: hop aroma intensity and quality; flavor fullness; bitterness quality; and overall preference. Radial neural networks were used to approximate the results of the study. Optimal process parameters were determined: Saphir hops, process duration 12 hours, temperature $3,0^{\circ} \mathrm{C}$. The optimum process duration is $10,5 \mathrm{~h}$ and the optimum temperature is $3,0^{\circ} \mathrm{C}$. Processing temperatures below $3.0^{\circ} \mathrm{C}$ result in a slightly lower optimization criterion than the $3.0^{\circ} \mathrm{C}$ processing temperature. At temperatures of 1.5 and $2.0^{\circ} \mathrm{C}$ the optimum process duration is 8.15 and 11.25 days respectively. The use of the combination of Saphir \& Chinook hops offers no improvement in the overall optimality criterion compared with the use of Saphir alone. The process duration of 4 hours achieves an optimization criterion of more than 0.60 , but only with durations of around 12 hours does an optimization criterion of more than 0.65 become attainable.
\end{abstract}

Keywords: hops, neural networks, Saphire, Chinook, optimization criterion, dry hopping

\section{References}

Borodulin, D. M., Safonova, E. A., Prosin, M. V., \& Milen'kii, I. O. (2017). Issledovanie protsessa okhmeleniya pivnogo susla s primeneniem sovremennogo oborudovaniya [Investigation of the process of hopping beer wort with the use of modern equipment]. Sovremennye materialy, tekhnika i tekhnologii [Modern Materials, Equipment and Technologies], 3, 16-21.

Chesnokova, A. N. (2011). Fenol'nye soedineniya khmelya obyknovennogo (Humulus Lupulus L.) i khmeleproduktov [Candidate Dissertation, Irkutskii go- sudarstvennyi tekhnicheskii universitet]. Irkutsk, Russia.

Fedorenko, B. N., \& Trenkl, F. (2017). Innovatsionnoe tekhnologicheskoe oborudovanie dlya kraftovogo pivovareniya [Innovative process equipment for craft brewing]. Pivo i napitki [Beer and drink], 1, 22-24.

Gernet, M. V. (2017). Perspektivy rasshireniya assortimenta napitkov brozheniya dlya pivovarennykh zavodov maloi moshchnosti [Prospects for expanding the range of fermentation drinks for small capacity breweries]. Pivo i napitki [Beer and Beverages ], 3, 14-17.

Grachev, Yu. P., \& Plaksin, Yu. M. (2005). Matematicheskie metody planirovaniya eksperimentov 
[Mathematical methods for planning experiments]. Moscow: DeLi print.

Korostelev, A. V., Rukavitsyn, P. V., Novikova, I. V., Kuchmenko, T. A., Umarkhanov, R. U., \& Murav'ev, A. S. (2020). Issledovanie aromatobrazuyushchikh komponentov khmelya s primeneniem khimicheskikh sensorov [Study of aroma-forming components of hops using chemical sensors]. Izvestiya Vuzov. Prikladnaya Khimiya $i$ Biotekhnologiya [Proceedings of Universities. Applied Chemistry \& Biotechnology], 10(3), 479486. https://doi.org/10.21285/2227-2925-202010-3-479-486

Matveeva, N. A., \& Titov, A. A. (2014). Vybor sorta khmelya dlya tekhnologii sukhogo okhmeleniya [Selection of hop variety for dry hop technology]. Nauchnyi zhurnal NIU ITMO. Protsessy i apparaty pishchevykh proizvodstv [Scientific journal of ITMO University. Processes and apparatus for food production], 4, 120-125.

Matveeva, N. A., \& Titov, A. A. (2015). Primenenie tekhnologii sukhogo okhmeleniya $\mathrm{v}$ pivovarenii [Application of dry hops technology in brewing]. Nauchnyi zhurnal NIU ITMO. Protsessy i apparaty pishchevykh proizvodstv [Scientific journal of ITMO University. Processes and apparatus for food production], 1, 111-118.

Meledina, T. V. (2003). Syr'e i vspomogatel'nye materialy $v$ pivovarenii [Raw materials and auxiliary materials in brewing]. S-Petersburg: Professiya.

Novikova, I. V., Agafonov, G. V., Korotkikh, E. A., Korostelev, A. V., Rukavitsyn, P. V., \& Novikov, S. V. (2018). Razrabotka metodiki proektirovaniya bezalkogol'nykh napitkov s uchetom sbalansirovannosti sostava [Development of design methodology for non-alcoholic beverages taking into account the balance of composition]. Pivo i napitki [Beer and Beverages], 2, 26-30.

Novikova, I. V., Rukavitsyn, P. V., \& Murav'ev, A. S. (2018). Vliyanie osnovnykh parametrov protsessa sukhogo okhmeleniya na fiziko-khimicheskie pokazateli piva [Influence of the main parameters of the dry hops process on physico-chemical parameters of beer]. Tekhnologiya $i$ tovarovedenie innovatsionnykh pishchevykh produktov [Technology and Merchandising of Innovative Foods], 6, 9-17.

Novikova, I. V., Rukavitsyn, P. V., \& Murav'ev, A. S. (2019). K voprosu perekhoda aromaticheskikh soedinenii khmelya $\mathrm{v}$ pivo pri realizatsii sukhogo okhmeleniya [On the transition of hop aromatic compounds into beer during the implementation of dry hops]. Pishchevaya promyshlennost' [Food Industry], 1, 69-73.

Osovskii, S. (2004). Neironnye seti dlya obrabotki informatsii [Neural networks for information processing]. Moscow: Finansy i statistika.
Shelekhova, N. V., Shelekhova, T. M., Skvortsova, L. I., \& Poltavskaya, N. V. (2021). Identifikatsiya spirtnykh napitkov s primeneniem iskusstvennykh neironnykh setei [Identification of alcoholic beverages using artificial neural networks]. Pishchevaya promyshlennost' [Food Industry], 3, 43-48. https:// doi.org/10.24412/0235-2486-2021-3-0028

Barry, S., Muggah, E. M., McSweeney, M. B., \& Walker, S. (2018). A preliminary investigation into differences in hops' aroma attributes. International Journal of Food Science \& Technology, 53(3), 804811. https://doi.org/10.1111/ijfs.13656

Bishop, C. M. (1995). Neural networks for pattern recognition. Clarendon Press.

Cibaka, M.-L. K., Ferreira, C. S., Decourrière, L., Lorenzo-Alonso, C.-J., Bodart, E., \& Collin, S. (2018). Dry hopping with the dual-purpose varieties amarillo, citra, hallertau blanc, mosaic, and sorachi ace: Minor contribution of hop terpenol glucosides to beer flavors. Journal of the American Society of Brewing Chemists, 75(2), 122-129. https:// doi.org/10.1094/ASBCJ-2017-2257-01

Donaldson, B. R., Bamforth, C. W., \& Heymann, H. (2012). Sensory descriptive analysis and freechoice profiling of thirteen hop varieties as whole cones and after dry hopping of beer. Journal of the American Society of Brewing Chemists, 70(3), 176181. https://doi.org/10.1094/ASBCJ-2012-0710-01

Dresel, M., Praet, T., van Opstaele, F., van Holle, A., Naudts, D., Keukeleire, D. de, Cooman, L. de, \& Aerts, G. (2015). Comparison of the analytical profiles of volatiles in single-hopped worts and beers as a function of the hop variety. BrewingScience, 68(1-2), 8-28.

Hao, J., Dong, J., Yin, H., Yan, P., Ting, P. L., Li, Q., Tao, X., Yu, J., Chen, H., \& Li, M. (2018). Optimum method of analyzing hop derived aroma compounds in beer by headspace solid-phase microextraction (SPME) with GC/MS and their evolutions during chinese lager brewing process. Journal of the American Society of Brewing Chemists, 72(4), 261-270. https://doi.org/10.1094/ ASBCJ-2014-1021-01

Haseleu, G., Intelmann, D., \& Hofmann, T. (2009). Identification and RP-HPLC-ESI-MS/MS quantitation of bitter-tasting beta-acid transformation products in beer. Journal of Agricultural and Food Chemistry, 57(16), 7480-7489. https://doi. org/10.1021/jf901759y

Kippensberger, M., Hanke, S., Biendl, M., Stettner, G., \& Lagemann, A. (2014). Transfer of nitrate and various pesticides into beer during dry hopping. BrewingScience, 67, 1-9.

Leitao, C., Marchioni, E., Bergaentzlé, M., Zhao, M., Didierjean, L., Taidi, B., \& Ennahar, S. (2011). Effects of processing steps on the phenolic content and antioxidant activity of beer. Journal of 
Agricultural and Food Chemistry, 59(4), 1249-1255. Van Opstaele, F., de Rouck, G., de Clippeleer, J., https://doi.org/10.1021/jf104094c

Maye, J. P., Smith, R., \& Leker, J. (2016). Humulinone formation in hops and hop pellets and its implications for dry hopped beers. Technical Quarterly, 53(1), 23-27. https://doi.org/10.1094/TQ-53-1-0227-01

Schönberger, C., \& Kostelecky, T. (2011). 125th Anniversary review. The role of hops in brewing. Journal of the Institute of Brewing, 117(3), 259-267. https://doi.org/10.1002/j.2050-0416.2011.tb00471.x

Takoi, K., Tokita, K., Sanekata, A., Usami, Y., Itoga, Y., Koie, K., Matsumoto, I., \& Nakayama, Y. (2016). Varietal difference of hop-derived flavour compounds in late-hopped/dry-hopped beers. BrewingScience, 69, 1-7.

Titus, B. M., Lerno, L. A., Beaver, J. W., Byrnes, N. K. Heymann, H., \& Oberholster, A. (2021). Impact of dry hopping on beer flavor stability. Foods, 10(6), 1264. https://doi.org/10.3390/foods 10061264 de Cooman, L. (2010). Analytical and Sensory Assessment of Hoppy Aroma and Bitterness of Conventionally Hopped and Advanced Hopped Pilsner Beers. Journal of the Institute of Brewing, 116(4), 445-458. https://doi. org/10.1002/j.2050-0416.2010.tb00796.x

Walsh, D. B., O’Neal, S. D., George, A. E., Groenendale, D. P., Henderson, R. E., Groenendale, G. M., \& Hengel, M. J. (2018). Evaluation of pesticide residues from conventional, organic, and nontreated hops on conventionally hopped, late-hopped, and wet-hopped beers. Journal of the American Society of Brewing Chemists, 74(1), 53-56. https://doi. org/10.1094/ASBCJ-2016-1115-02

Zanoli, P., \& Zavatti, M. (2008). Pharmacognostic and pharmacological profile of Humulus lupulus L. Journal of Ethnopharmacology, 116(3), 383-396. https://doi.org/10.1016/j.jep.2008.01.011 